\title{
REPORT: LGBT+ FACULTY RETENTION IN STEM DISCIPLINES AT POSTSECONDARY INSTITUTIONS IN

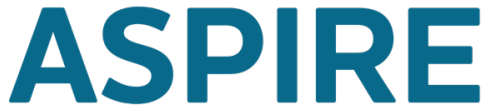 \\ THE UNITED STATES
}

THE NATIONAL ALLIANCE FOR INCLUSIVE \& DIVERSE STEM FACULTY

\author{
Alyssa Ryan, Aspire Graduate Fellow
}

A vast majority of retention efforts to increase faculty diversity have focused on race and gender with little to no attention paid on Lesbian, Gay, Bisexual, Transgender, plus (LGBT+) communities ${ }^{1}$. The extensive literature focused on women faculty and faculty of color does not encapsulate the spectrum of experiences for LGBT+ faculty. Diverse faculty enhance the education for all students and encourage student persistence for underrepresented minority students. While there is significant research on LGBT+ faculty in the social sciences, humanities, and health fields however, LGBT+ communities in STEM disciplines have not been studied widely².

One overriding challenge LGBT+ STEM faculty may face is the persistent belief that LGBT+ issues are irrelevant to both the disciplinary perspectives in STEM and that the "objective" nature of STEM means that identity does not factor into how scholars and students are treated 3 . Research has shown acceptance of LGBT + faculty and students is dependent on discipline with STEM disciplines being the least welcoming and the social sciences the most welcoming. LGBT+ faculty have reported experiencing more hostility than colleagues and more pressure to be their inauthentic selves-covering their sexuality and gender identity becomes a necessity ${ }^{2}$. Adding to this, "many faculty members, especially faculty of color, conducting scholarship on or related to their specific self-identity may feel devalued within the

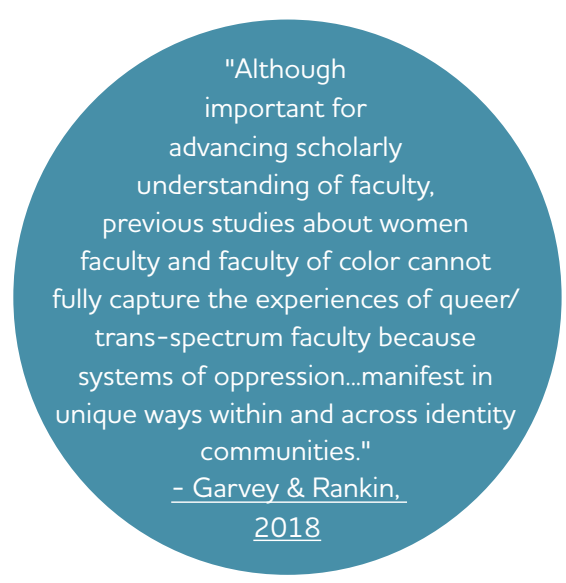
academy"3; this extends to LGBT+ faculty as well.

Studies have found that LGBT+ faculty in STEM are more likely to leave or consider leaving their institutions due to feelings of oppression and hostility amongst members of the department including staff, faculty, and students ${ }^{4}$. In addition, for LGBT+ faculty, the dilemma is whether to be open (out) about their sexuality and/or gender identity or not. While STEM disciplines report to have a higher percentage of out faculty than other disciplines, heterosexual faculty experience a freedom LGBT+ faculty do not-talking about their identities, experiences, or partners openly without fear of repercussion from students, colleagues, or administration. Faculty who do choose to be out in their academic community may see an increase in service responsibilities related to their LGBT+ identities.

Being public about one's sexuality for LGBT+ faculty may lead to lower teaching evaluations, lower course enrollments, and microaggressions. Presumptions of heterosexuality and other heteronormative assumptions of colleagues silence any conversations regarding sexuality and non-binary gender identities in STEM workplaces 5 . The "pressure to conform or conceal aspects of identity is felt much more intensely by those whose identities fall farther outside the stereotype of a straight, cisgender, white, middle class, man" 5 . In addition to (micro)aggressions regarding their gender identity or sexuality, faculty of color who also identify as LGBT+ may face compounding issues related to racism or xenophobia. 
The scant research-based information available about LGBT+ faculty limits recommendations on how universities and STEM departments can and should support this population of faculty. Institutions should evaluate their campus climates to ensure safe and welcoming environments for all URG faculty including LGBT+ faculty. Understanding the experiences of the LGBT+ faculty on campus will be essential for crafting educational and programmatic support. Since many institutions do not collect demographic data about their LGBT+ faculty, climate and satisfaction studies may be the only way for institutions to understand this often invisible population on campus.

Institutional leaders should also increase the educational resources on gender identity and sexuality available to faculty and administrators, with a particular focus on how to support LGBT+ colleagues -- not just students. These resources should incorporate information on the queer and trans spectrums and ensure a multi-faceted, rather than monolithic, view of diversity within LGBT+ communities. Ideally, these resources would move beyond whiteness and ableness frameworks to include information on intersectional identities that may create additional implicit or explicit (micro)aggressions. These resources must then be integrated into training and other public forums acknowledging LGBT+ faculty as members of the university and department community.

Institutions must also find ways to acknowledge and reward additional service responsibilities. The onus should not solely be on LGBT+ faculty to provide support, perform service, educate department faculty, staff, and students, and/or develop programming for the department's LGBT+ community. Institutions and departments should invest appropriately in developing a range of DEl-informed practices that actively incorporate, include, and value the perspectives and experiences of LGBT+ and other URG faculty in the development of initiatives, policies, and programming, while not placing the entire burden of that work on those faculty. It is then the responsibility of the department to include and value any additional service work performed by LGBT+ faculty to support these efforts during the promotion and tenure process.

1. LGBT+ is used to emphasize sexuality and gender identity-based cultures are diverse. The term is not exclusive to those who identify as lesbian, gay, bisexual, or transgender; rather, it may be used to refer to anyone who is non-heterosexual or noncisgender.

2. Patridge, E.V., Barthelemy, R.S., \& Rankin, S.R. (2014). Factors impacting the academic climate for LGBQ STEM faculty. Journal of Women and Minorities in Science and Engineering. 20(1), 75-98.

3. Linley, J.L. \& Nguyen, D.J. (2015). LGBTQ experiences in curricular contexts. New Directions for Student Services. (152), 41-53.

4. Garvey, J.C., Rankin, S.R. (2018). The influence of campus climate and urbanization on queer-spectrum and trans-spectrum faculty intent to leave. Journal of Diversity in Higher Education, 11(1), 67-81.

5. Mattheis, A. , Cruz-Ramierz De Areelano, D., \& Yoder, J. B. (2019). A model of queer STEM identity in the workplace. Journal of Homosexuality, DOI: 10.1080/00918369.2019.1610632.

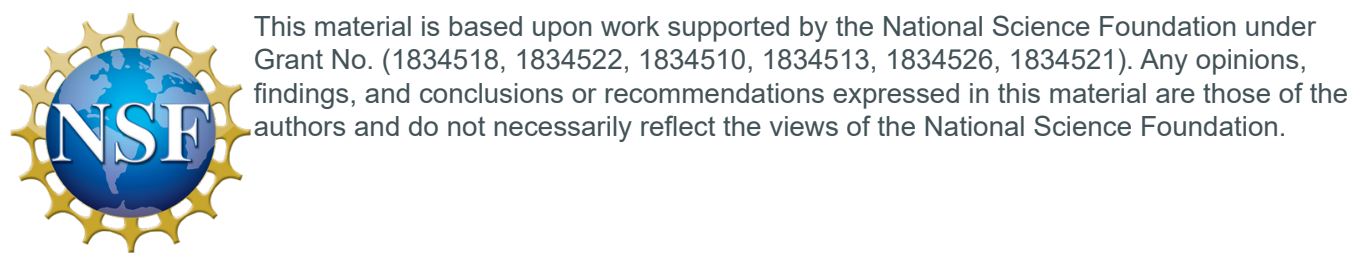

